\title{
Nanostructure of synthetic Mn- stannite microspheres as revealed by TEM
}

EDYTA WALUŚ AND MACIEJ MANECKI

AGH University of Science and Technology

Presenting Author: ewalus@agh.edu.pl

Copper-based quaternary chalcogenides have been deeply studied in the past decade due to their low cost and potential high photovoltaic efficiency. Mn-stannite $\mathrm{Cu}_{2} \mathrm{MnSnS}_{4}$ (CMTS) shares several properties with the widely investigated $\mathrm{Cu}_{2} \mathrm{ZnSnS}_{4}$ (CZTS) for photovotovoltaic, catalysis, thermoelectrics, spintronics, supercapacitors or serves as electrodes in batteries applications. We report the results of hydrothermal synthesis of CMTS structures and discuss the effect of different sulfur precursors on their growth, properties and relations in the mechanism of formation. Inorganic sodium sulfide $\left(\mathrm{Na}_{2} \mathrm{~S}\right)$ and organic tiourea $(\mathrm{Tu})$ were used as sulfur source. Scanning electron microscopy (SEM) and transmission electron microscopy (TEM) were used to characterize the morphology. Structural properties were determined by X-ray diffraction (XRD) and electron diffraction (TEM/SAED selected area electron diffraction and TEM/EBSD electron backscatter diffraction). Raman spectroscopy was used to identify impurities in the synthesis material. When TU is used as a sulfur source, CMTS precipitates in the form of concentric microspheres composed of hexagonal forms in the core and tetragonal forms in the microsphere rim. For the first time, the mutual relationship of these structures was revealed. The presence of hexagonal forms in the nucleus of microspheres indicates their rapid formation during the initial stages of synthesis. Subsequently, nanometersized spherical aggregates are surrounded by a rim of slowerforming tetragonal nanocrystals. This is consistent with an earlier report by Zou et al. (2013) that the type of sulfur precursor influences the structure of the resulting product through different reactivity of the metal with sulfur. These authors also predicted theoretically that such an effect could be observed for Mnstannite, which was experimentally confirmed in this work.

This work is partially funded by Polish budget funds for science in 2017 - 2019 as a research project No DI2016004946 under "Diamond Grant" program. 